\title{
IMIQUIMOD - A TOLL LIKE RECEPTOR 7 AGONIST - IS AN IDEAL OPTION FOR MANAGEMENT OF COVID 19
}

Konstantinos Poulas ${ }^{1}$

1 University of Patras

\section{Abstract}

According to numerous recent publications, the COVID-19 patients have lymphopenia, higher infection-related biomarkers and several elevated inflammatory cytokines (i.e. tumor necrosis factor (TNF)- $\alpha$, interleukin IL-2R and IL-6). The total number of B cells, T cells and NK cells are significantly decreased. RNA viruses, SARS-CoV-2 included, hit the innate immune system in order to cause infection, through TLRs 3, 7 and 8. Imiquimod is an immune-stimulator that activates TLR 7 and can be used to enhance the innate and adaptive immunity. Preclinical and clinical trials are proposed.

Athina Angelopoulou, Nikos Alexandris, Evangelia Konstantinou, Konstantinos Mesiakaris, Konstantinos Farsalinos, Charilaos Zanidis and Konstantinos Poulas

Laboratory of Molecular Biology and Immunology, Department of Pharmacy, University of Patras, 26500, GREECE

SUMMARY

Coronaviruses (CoVs) are classified to a large family of positive-sense single stranded RNA viruses ((+)SSRNA) in the genus of beta-coronaviruses that exploit their genetic material, after internalization in the host cells, to act as messenger RNA, so as to be translated to viral structural proteins, necessary for replication and transcription of the viruses.(1) CoVs are greatly pathogenic in humans triggering respiratory infections ranging from the common cold to more severe diseases, like Middle East Respiratory Syndrome (MERS) and Severe Acute Respiratory Syndrome (SARS). The virion of CoVs is of "core-shell" morphology illustrated as viral envelopes, with the viral CoVs' RNA genome being protected, in the core, inside nucleocapsids of protein origin and helical 
symmetry that are enveloped in an outer shell of lipidic membrane orig in (lipid bilayer), typically deriving from the cellular membranes of the host cells. The viral envelope is of phospholipidic, proteinic and glycoproteinic orig in and may serve as a kamuflaz to escape the immune system of the host, and may exploit its surface glycoproteins to identify and link to receptors on the cellular host membrane. $(1,2)$ CoVs belong to the RNA viruses with increased prospective of becoming pandemic worldwide. The 2019 novel coronavirus, called 'SARS-CoV-2' is a new strain that causes Corona Virus Disease 2019 (COVID-19), for which no effective treatment has been found until now. The outbreak of SARS-CoV-2, that first emerged in Wuhan in December 2019, has rapidly spread throug hout the world.(2) Considering the ong oing outbreak in China and fast worldwide spread of COVID-19, infected by SARS-CoV-2, it has led to the declaration of Public Health Emergency of International Concern by the World Health Organization (WHO) on 30 January 2020.7 As of Apr 10, 2020, a total of more than 1,500,000 of laboratoryconfirmed new cases of COVID-19 has been identified in the world with more than 89,000 deaths.

In order to deal with COVID-19 pandemic supportive and preventive therapeutic approaches have been followed with directives and guidelines of social distancing and isolation worldwide, while scientists, and researchers work in order to elucidate the clinical spectrum of COVID-19, the transmission mechanisms, the virus-host interactions, and seek new diagnostic, preventive (vaccines) and therapeutic approaches. In this short review, the basic molecular replication mechanism of SARS-CoV-2 and the consequences of the imminent cytokine storm on host cells are outlined on the base of CoVs immune elusion and host cells innate immunity, which represent key points for effective defense against COVID-19 infection. The drug Imiquimod a synthetic molecule able to enhance both the innate and acquired immune response is proposed as an effective therapeutic approach.

\section{IMMUNOLOGICAL DATA}

How Coronavirus Replicate in the Host Cell?

Extensive studies have been researched in the field of etiology, epidemiology and pathophysiology of CoVs in relation to COVID-19.(3,4) In CoVs generally, the spike S glycoproteins (composed of S1 and S2 subunits) on the surface of the viral envelop guide the receptor mediated endocytosis by attachment to host cell receptors of AngiotensinConverting Enzyme 2 (ACE2) for SARS-CoV and Dipeptidyl Peptidase 4 (DPP4) for MERS- 
CoV, respectively. By this binding process, CoVs manage to escape from immune surveillance, and thus host cellular membrane fusion is promoted, wherein the viral genome is released in the cytoplasm in order to be translated in the ribosomes of the host cell.(3) Then the released viral genome in the host ribosome, uses the open reading frames (ORFs), especially the ORF1 $a$ and $1 \mathrm{~b}$, to be translated to long polyproteins $1 \mathrm{a} / 1 \mathrm{ab}$ (pp1a/pp1ab). Polyproteins are further processed by viral encoded proteases (such as, chymotrypsin-like protease 3CLpro, main Mpro protease and papain-like proteases) for the final product of 16 non-structural proteins (nsps 1-16).(5) The main role of nsps is to prepare a favorable cellular environment and enhance viral replication (nsps1), affect viral growth kinetics (nsps2), affect viral fitness, active-site mutants and conformational changes (nsps3-5), affect non-specific RNA-binding activity (nsps 7-10), contain the RNA replication and transcription enzymes (nsps12-16). Especially, in nsp12 the coronavirus RNA-dependent RNA polymerase is contained.(6) According to Knoops et al., (6) the transmembrane nsps are probably inserted into the endoplasmic reticulum (ER) membranes, where early viral RNA synthesis possibly occurs. At the time of increased replication expression being achieved, nsps promote membrane pairing and bending for membrane envelopes to be formed.

Even if CoVs RNA synthesis is incompletely understood, the viral genomic RNA following translation into polyproteins in accordance with structural (S (stripe protein, $\mathrm{M}$ (membrane protein), N (nucleocapsid protein), E (envelope protein) and non-structural proteins, serves as template for the synthesis of negative sense RNA species and smaller species of subgenomic RNAs (sgRNAs) sequences of both polarities. The positive sense sgRNAs are received as messages for all ORFs expression. The viral genome is mainly acting as a loop template for replication and transcription. The replicated positive sense sgRNAs become the progeny genomes, where the viral $\mathrm{N}$ structural protein is directed to bind and form newly developed nucleocapsids for the progeny virions. Following the secretory pathway, the new nucleocapsid along with viral structural proteins S, E, and M penetrate into the ER or Golgi intermediate compartment, wherein through proteinprotein interactions progeny virion assemblies with incorporated nucleocapsid are formed. Finally, the progeny viruses transported by Golgi vesicles penetrate into the ER lumen and after incorporating its lipid bilayer membrane, they are directed to the cellular membrane, in order to be exocytosed into the extracellular environment able to infect other host cells. $(5,6)$

Does Cytokine Storm affect the outcome of Coronavirus Infection? 
Unfortunately, a thorough research study on the virus-host interactions in case of SARSCoV-2 during respiratory infection is yet limited, thus the available information arises from investigation on other RNA CoVs, such as SARS-CoV and MERS-CoV or for recent clinical date on COVID-19. According to numerous publications,2 the most well recognized hematologic abnormality for the patients is lymphopenia that in severe cases tend to be up to $85 \%$ (increasing rate with severity of the cases), higher infection-related biomarkers and elevated levels of several pro-inflammatory cytokines (i.e. tumor necrosis factor (TNF)- $\alpha$, interleukin IL-2R and IL-6). In distinct report studies from China $(2,4,7,8)$ increased levels of neutrophils, c-reactive protein and IL-6, accompanied with low levels of lymphocytes were observed, while increased levels of innate proinflammatory cytokines, such as TNF- $\alpha$ and chemokines such as IP-10 (interferon- $\gamma$ inducible protein), MCP-1 (monocyte chemoattractant protein), MIP-1 $\alpha$ (macrophage inflammatory protein 1 alpha), were observed in patients needing intensive care unit (ICU) hospitalization. Higher serum levels of pro-inflammatory cytokines (TNF- $\alpha$, IL-1 and IL-6) and chemokines (IL-8) were also found in patients with severe COVID-19 compared to individuals with mild disease. The elevated plasma levels of neutrophils, pro-inflammatory cytokines and chemokines and the decrease on lymphocyte levels have been correlated with disease severity and mortality.(8)

The intense and uncontrolled release of pro-inflammatory cytokines has been associated to cytokine storm (CS), a syndrome that can be promoted by infectious diseases, such as COVID-19, resulting in systemic inflammation, acute lung injury, acute respiratory distress syndrome (ARDS), multiple organ failure (especially acute kidney injury, cardiac injury, spleen atrophy, lymph node atrophy) 10 and death.(7-9) One of the foremost and most severe consequences of SARS-CoV-2 is ARDS, an immunophathological in patients. In Huang et al. (5) report, based on patients hospitalized in Wuhan with early stage symptoms of COVID-19, 26 out of 41 (63\%) patients developed lymphopenia and 12 of 41 (29\%) suffered of ARDS, while 13 (32\%) patients were admitted to an ICU and 6 (15\% of 41 patients) died. All patients hospitalized in ICU presented cytokine storm syndrome with elevated levels on plasma serum of pro-inflammatory cytokines and chemokines, such as IL-7, IL-10, GSCF, IP-10, MCP-1, MIP-1 $\alpha$, and TNF $\alpha$. Even if the pathophysiology of COVID-19 is not fully understood yet, the presented cytokine storm has been related to disease severity. (7-10) The elevated secretion levels of pro-inflammatory cytokines in CoV infections, such as IL-6, IL-12, IFNy, IP-10, MCP-1 has thoug ht to possibly promote the activation of T-helper-1 (Th1) cell responses, while the GCSF, IP-10, MCP-1, MIP-1 $\alpha$, and TNF $\alpha$ pro-inflammatory chemokine storm has been associated to a possible promotion of T-helper-2 (Th2) cell responses.2 In the 2019 inflection of SARS-CoV-2 
elevated secretion levels of cytokines related with Th1 responses and forthcoming pulmonary inflammation, and cytokines of Th2 responses associated with suppression of inflammation, have been reported according to the stage of the disease. (11)

Are Immune Responses and Innate Immunity the key for COVID-19?

SARS-CoV-2 infection has been related to lymphocytopenia with decreased levels of lymphocytes ( $T$ cells, B cells and natural killer cells, NK) being correlated with disease severity in most patients. Previous studies on MERS-CoV and SARS-CoV infections have identified that $T$ cells especially $C D 4+$ and $C D 8+T$ cells, play a significant antiviral role. (12) In recent reports on the clinical features, the total number of B cells, T cells and NK cells significantly decreased in patients with COVID-19 and more evident in the severe cases, compared to the non-severe group.(8-11) Especially, T cells were shown to be more affected by SARS-CoV-2, as T cell count was nearly half the lower reference limit. The function of CD4+, CD8+ T cells, and NK cells was within normal range and no significant difference was found between severe cases and non-severe ones.(8) In viral infections, the response of the host immune system after virus invasion is to recognize and control the infectious DNA or RNA genome released in the host cells cytoplasm. CoV infections can cause uncontrolled immune responses, through the cytokine storm syndrome and result in immunopathogenesis in the host. In particular, SARS-CoV infections the S protein on the virion corona is linked to the ACE2 receptor of the host cell and after fusion via the cytoplasmic membrane, the viral RNA genome is released into the cytoplasm. Then, normally the innate immune response signaling cascade starts with the recognition of the viral genome, representing a pathogen-associated molecular pattern (PAMP), by the pattern recognition receptors (PRRs), which in general are proteins responsible for detecting pathogenic stimuli. It is well known that the innate immune system is important in early life, when the adaptive functions are underdeveloped. At present, the response of the innate immune through the PRRs is the first line of defense against viral infections, thus PRRs, such as toll-like (TLR), RIG-I-like (retinoic acid-inducible gene-I-like receptors, RLR), NOD-like (nucleotide-binding oligomerization domain-like receptors, NLR), C-type lectin-like (CLRs), should provide robust and efficiently synchronized effect. $(13,14)$

However, when immune response is dysregulated it will result in an excessive inflammation, even cause death. Qin et al. demonstrated pronounced lymphopenia and low counts of CD3+ and CD4+ cells in COVID-19 cases.(8) Similar clinical features are observed in SARS-CoV with patients of severe groups after convalescent indicating 
polyfunctional CD4+ T cells producing IFN- $\gamma, T N F-\alpha$, and IL-2, and CD8+ T cells producing IFN- $\gamma$, TNF- $\alpha$, and CD107a and elevated immune responses of Th2 cytokines (IL-4, IL-5, IL-10), while in MERS-CoV patients of increased severity elevated CD8+ T cell levels were observed with CD4+ T cell responses being minimal. In the latter case after convalescence severity-dependent antibody responses and antigen-reactive cells were observed.(8) From the clinical findings, it is considered that T cell responses are clearly related to CoVs severity. In the lungs usually, PAMPs in the form of genomic RNA viruses or dsRNA intermediates are recognized, during replication in the host, by the Toll-like receptors (TLRs) 3, 7, 8, and endosomal RNA receptors or cytoplasmic RNA PRRs, such as RIG-I (retinoic-acid inducible gene I, also known as DDX58) and MDA5 (melanoma differentiation-associated 5) representing highly important receptors, since they provide first line defense against infections.(11-14) The attachment of viral genome in the receptors give rise to innate immune response signaling pathways. Especially, for TLRs expressed on the membranes of leukocytes (i.e. immune cells, such as dendritic cells, macrophages, natural killer cells, cells of the adaptive immunity T cells, and B cells) the attachment of viral RNA triggers molecular cascades of innate immune responses and development of acquired antigen-specific immunity. The activation of TLRs and the involvement of T cell antigen receptors (TCR) promotes critical signal transduction cascades through the activation of signal transducing adapter proteins (STAPs), such as MYD88, to transfer the antigen-induced signal transduction pathway. A series of molecular events is involved in signal transduction, usually protein phosphorylation catalyzed by protein kinases (IKKi, IRAK1, IRAK4, and TBK1) resulting in cytokine production and innate immune responses.(11-14) Moreover, in viral infections the innate immune system senses foreign material that is possibly pathogenic, and this triggers downstream signaling to ultimately induce transcription factors in the nucleus, which in turn stimulate expression of types I and III IFNs and other pro-inflammatory cytokines. (11-14) Once released, type I IFNs bind to IFN- $\alpha / \beta$ receptor (IFNAR) on viral cells resulting in the activation of JAK-STAT signaling pathway. In a sequence of molecular events induced by IFNs action, encountering enzyme production (protein kinase R, RNAse L), and protein phosphorylation, the viral and host RNA genome within the cells are destroyed, in order to reduce virus and infected cells production and replication.(11-14) Although, CoVs are highly sensitive to IFNARs the SARS-COV and MERS-CoV viruses remain extremely pathogenic, possibly since the $\mathrm{N}$ structural proteins of CoVs employ tactics of immune escape proteins in order to serve antagonistically against host IFN response.(15)

Generally, IFN induce the production and transcription of IFN-stimulated genes (ISGs) under the control of IFN-stimulated response element (ISRE), with the action of 
prevention and suppression of viral replication, particularly at an early stage of the infection.(3) Moreover, autocrine signaling induces the secretion of hormones or autocrine messenger agents, such as IL-1, IL-2, IL-6, in the host cell, which attach to autocrine receptors leading to stimulation of genes inside the host cell itself. Alternatively, paracrine signaling promotes secretion of paracrine factors, which diffuse extracellularly, in the relatively close environment, to diffuse to nearby cells and bind to paracrine receptors, where signal transduction cascades are initiated. Mainly, there are four families of paracrine receptors and consequent signaling pathways the fibroblast growth factor family (FGF with receptor tyrosine kinase pathway and JAK-STAT pathway), Hedgehog family, Wnt family, and T GF- $\beta$ superfamily. Subsequently, a second round of autocrine and paracrine signalling ensures that infected, and the surrounding uninfected cells, express a myriad of IFN-stimulated genes that establish a so-called antiviral protection state.(15) The strict distinction between innate and adaptive ( $T$ cells, CD4+ T cells, and CD8+ T cells, B cells) immune responses is probably not accurate. In the respiratory tract, several cell types and mechanisms that integ rate aspects from both branches of human immunity are thought to be very important for the defence against respiratory infections. NKs, T cells, mucosal-associated invariant T cells, and neutrophils, form a bridge between the innate and adaptive machineries and play very important roles during the clearance of respiratory viruses.(15)

In overall, the role of type I IFN is vital in respiratory CoV infections, especially in SARSCOV and MERS-CoV cases with increased mortality. Both type of CoVs promote an associated disease severity by utilizing their structural proteins and ORFs of the nonstructural proteins to meddle in the host cells' signalling pathways and at the same time downregulate signalling receptors, such as IFN receptors. This type of interfering leads to pulmonary and systemic inflammatory responses with elevated levels of leukocytes, including lymphocytes, neutrophils and monocytes/macrophages.(8-12,15) SARS-CoV-2 genomic sequence presents similarities with SARS-CoV and MERS-CoV genomes, thus it is considered that upon COVID-19 infection the low levels of pro-inflammatory cytokines, leukocytes and type I IFN is related to early disease stage, however as infection severity increases cytokine storm and elevated levels of type I IFN leads to pulmonary immunophathological syndromes, such as pneumonia and ARDS. Unfortunately, a protective vaccine against CoVs that would provide essential protection ag ainst these infections has not been developed yet, even if is highly researched. Thus, effective therapeutic measures need to be researched to eliminate the virus, taking advantage of the insight on the innate immune system, at an early stage for valuable immunological responses, wherein CoVs infections can be controlled efficiently with least pulmonary 
immunological consequences.

\section{IMIQUIMOD AS AN IMMUNOSTIMULATOR AND SOLUTION TO COVID-19}

How Imiquimod effect on the Immune System is developed?

Imiquimod ((IMQ) is a non-nucleoside heterocyclic amine which belongs to the class of $1 \mathrm{H}$-imidazo-[4,5-c] quinolones.(16) The precise mechanism of action of this synthetic molecule is unknown. However, preclinical studies revealed that imiquimod modifies the immune response by enhancing both the innate and adaptive immune system, in particular the cell-mediated pathways. Generally, imiquimod acts as an immune response modifier, in an indirect manner, as it induces immune reactions and the secretion of many cytokines, which in turn stimulate T cells. $(16,17)$ Moreover, it has been used as a potent antiviral and antitumor agent in different animal models. $(16,17)$

Innate immune system is based on the recognition of pathogens from the organism and activation of many cell types, which eliminate them. Imiquimod exerts its action in innate immune system by binding to cell surface receptors, such as toll like receptors (TLRs). There are 10 types of TLRs that recognize microorganisms or specific components derived from pathogens. Imiquimod exerts its biological action through TLR-7 and TLR8.18 This interaction leads to activation of a signaling cascade, which promotes translocation of nuclear factor-kappa B (NF-kB). NF-kB binds to DNA and induces the expression of many pro-inflammatory cytokines from the peripheral blood mononuclear cells, such as interferon- $\alpha$ (IFN- $\alpha$ ), tumor necrosis factor $\alpha$ (TNF- $\alpha$ ), interleukin IL-1, IL-2, IL-6, IL-8, IL-12, granulocyte colony stimulating factor (GM-CSF), granulocyte macrophage colony stimulating factor (GM-CSF), as well as chemokines, such as CCL4 and CCL2.(16-18) The acute antiviral and antitumor effects of imiquimod are largely originating from its ability to induce innate immune responses, especially its ability to induce secretion of IFN- $\alpha$ and other cytokines, such as IL-6, IL-12 and T NF- $\alpha$, which has been observed in many studies.(16-19) Increased expression of IL-1 and IL-6 leads to the activation of T lymphocytes and IL-12, which is produced by macrophages, induces the production of IFN- $\gamma$ by natural killer (NK) and T cells. Furthermore, imiquimod activates antigen presenting cells, such as dendritic cells, macrophages activated to secrete both cytokines and nitric oxide and B lymphocytes activated to proliferate and differentiate, which in turn activate the adaptive system.(16) 
Another cell type that is activated and migrates towards the side of the infection, in response to IFN- $\alpha$, IFN- $\gamma$ and IL-12 are Langerhans cells, which in turn induce immune responses. These cells show increased mobility in the presence of imiquimod migrating to the regional lymph node and function as major antigen presenting cells.(16) Moreover, cytokines that are produced from innate immune responses, induce indirectly the production of IFN-Y from T helper cell type 1 (Th1), which are part of the adaptive immune system. IFN- $\alpha$ enhances the expression of IL-12 receptor B2 subunit on Th1 cells, which in turn respond to IL-2 and become the main source of IFN-Y. Moreover, IFN- $\gamma$ in combination with IL-2 produced from Th1 cells, activates CD8 cells that are converted to cytotoxic T cells, in order to eliminate cells infected from virus and to provide the immune memory, which is necessary for a future contamination.(16,19) Furthermore, IFN- $\gamma$ and IFN- $\alpha$ inhibit the production of cytokines IL-4 and IL-5 from Th2 cells. Overall, imiquimod has a unique mode of action as it does not directly kill the infected cells, but its strong antiviral and antitumor activity is due to its ability to enhance the production of many pro-inflammatory cytokines and to trigger an immune response. (16) The action of Imiquimod to stimulate innate immunity indicates its potential to treat viral infections.

Imiquimod application in Clinical Therapy: Examples of in vivo and clinical trials

Imiquimod (IMQ), an immune response modifier, has shown to have antiviral and antitumor attributes. Specifically, imiquimod induces (2'-5')-oligoadenylate synthetase, which confers an antiviral state and upregulates natural killer cells activity in vivo and in vitro. IMQ also enhances cell-mediated immunity. Lately, Nerurkar et al.(20) presented upregulated levels of chemokines in imiquimod treated mice presented three to five days after treatment. Fuertes et al.(21) observed a higher IMQ efficacy for the treatment of anal condylomas compared to anal HSIL in HIV-infected individuals.

In in vitro studies against human peripheral blood mononuclear cells (PBMCS), imiquimod at $1-5 \mu \mathrm{g} / \mathrm{ml}$ induces the production of several cytokines including several subtypes of IFN- $\alpha$, TNF- $\alpha$, IL-1, IL-1RA, IL-6, IL-8, IL-10, IL-12 p40, granulocyte colony stimulating factor (G-CSF), granulocyte/macrophage colony stimulating factor (GM-CSF) and macrophage inflammatory protein 1- $\alpha$ (MIP-1), MIP-1 $\beta$ and macrophage chemotactic protein (MCP-1) and at a low drug concentration about 0,5 $\mu \mathrm{g} / \mathrm{ml}$ is found that IFN-a and IL-1RA are the only cytokines increased.(22) Moreover, in in vivo studies that IMQ was administrated orally to mice (p.o.), stimulated a dose-dependent increase in serum levels of IFN- $\alpha$, being active with doses as low as $3 \mathrm{mg} / \mathrm{kg} .17$ IFN- $\alpha$ levels were detectable $1 \mathrm{~h}$ 
after treatment, with peak levels occurring at $2 \mathrm{~h}$ after treatment. Optimal dosage of imiquimod ranged between 10-100 mg/kg, with higher dosages (150-250 mg/kg) inducing similar secretion levels. Multi-dose regimens were also tested, separated by 2 hours, showing enhanced levels of IFN- $\alpha$. Multiple doses of imiquimod on the same day caused aug mented IFN- $\alpha$ levels, however high daily doses of IMQ to mice resulted in a hyporesponsive state characterized by reduced cytokine induction, while separation of the doses by four or more days caused normal levels of cytokine induction. In rats, oral administration of imiquimod, in doses $2 \mathrm{mg} / \mathrm{kg}$ or more, induced increased serum levels of IFN- $\alpha$ and TNF- $\alpha$ and the kinetics of induction were similar to those seen in mice induction.(17)

Very recently, a thought-provoking analysis was put forward, demonstrating the effect of IMQ against influenza A virus infection.(23) The authors provided with proof that delivery of imiquimod in mice, directly to the lungs via intranasal administration resulted in decreased expression in viral replication, airway inflammation, leukocytes levels (i.e. inflammatory cells such as macrophages, neutrophil, eosinophil), and pro-inflammatory cytokines/chemokines (IL-6, IFN- $\gamma$, IL-1 $\beta$, GCSF, CCL3, CXCL2, TNF- $\alpha$ )) following influenza A virus infection. The effect was actually stronger in intranasal administration compared to an epicutaneous one. The therapy resulted in a near 5 -fold elevation in Type I IFN- $\beta$ in the lung tissue of mice after 3 days of treatment. Moreover, imiquimod significantly suppressed the mRNA levels of IL-6, CCL3, CXCL2 and IL-1 $\beta$, otherwise increased in nonIMQ treated mice, whereas yielded a significantly higher antibody response in IgG1, IgG2a, IgE, IgM and total levels of IgG during infection. Additionally, TRL7 activation by IMQ was observed through a pronounced Type I IFN prompted response. Finally, triggered response of adaptive immune system was observed by efficient activation on the response of T lymphocytes (CD8+) and T helper cells (CD4+). In overall, IMQ effective treatment of influenza A virus was presented by suppression of inflammatory cells, cytokines/chemokines, activation of important type I IFN, and triggered adaptive system responses, while no significant lung dysfunction was observed as presented by tissue damping of naïve in comparison to influenza infected animals.(23) Imiquimod, a potent TLR7 agonist, could be a strong primer of the immune response to infectious pulmonary viruses, such as CoVs.

Numerous clinical trials present imiquimod as a potent and versatile compound that can enhance cellular activity and innate immunity.(24-26) Especially, clinical trials in humans either with topical treatment or with suppositories revealed prevention of recurrences in anal canal condyloma, whereas in infants with infantile hemangioma the moderation in 
surface erythema was notably faster than in prior experience.(24) Kreuter et al.,(25) further stressed the fact that application of imiquimod suppositories in intra-anal HPV Types 6 and 11 in HIV-Infected men after the surgical removal of intra-anal Condylomata Acuminata may yield better recurrences under the absence of sexual intercourse, while HIV-associated immunosuppression probably leads to a new increase in HPV-11 DNA load. Both Kaspari et al.(24) and Kreuter et al.(25) utilized 5\% imiquimod (in cream Aldara) in suppositories anally. IMQ due to its antiviral and anti-inflammatory action has also been researched in its role for the treatment of HPV in the development of cervical intraepithelial neoplasia (CIN). Specifically, in fifty-nine patients suffering from CIN an incremental administration was followed, in which administered dose was increased every two weeks until the maximum dosage of three vaginal suppositories (6.25 mg IMQ). CIN patients were divided in two groups one of placebo administration and the other of IMQ suppositories.(26) The IMQ group presented higher histologic reg ression and remission in correlation to the placebo one. A 60\% clearance of CIN was observed in the IMQ treated patients proving a well-established antiviral effect of imquimod against HPV. (26)

In other trials the topical application of IMQ was researched.(27-31) Actinic keratosis, a common cutaneous, pre-cancerous neoplasm appearing as rough, dry, scaly lesions that occur primarily on chronic ultraviolet (UV) light exposure on skin of middle-aged and elderly people is a field that researchers took interest in. Torres et al.(27) through a double-blind, placebo-controlled, randomized study in 17 patients presented an increased expression of TLR3, TLR7, and TLR8, consistent with increased expression observed in human peripheral blood mononuclear cells upon treatment with imiquimod. The induction of several members of the cytoplasmic helicase innate immune pathway, as well as several TLRs, indicates that in addition to activation of the TLR7 pathway treatment with IMQ also results in priming of other innate pathways, which may aug ment other aspects of the innate immune response. Moreover, it is also stated the fact that the increase of CD8ß, SELL, NT5E (CD73), LGALS2 (galectin 2), and LAIR1 (leukocyte-associated immunog lobulin-like receptor 1) receptors, as well as T-cell receptor (TCR) subunits TRD and TRG, TCR-signaling pathway genes (such as Fyn, Fyb and LCP2), genes associated with T-cell activation (such as HCK, CD69, PTPRC (CD45) SELL (CD62L, L-Selectin)), IT GA4 (antigen CD49D, alpha 4 subunit of VLA-4 receptor), and LAG3 are indicative of the stimulation of the adaptive immune system. Observations regarding the increase of $\mathrm{T}$ cell activation were also provided in a fifty-two patient study with topical treatment and the same administration by Seters et al.(28) 
patients with skin metastases was investigated. In this study, the variability of preexisting lymphocytic infiltrates within the cutaneous metastases and the lack of consistent quantitative changes of the infiltrate in biopsies were in contrast to the induction of a Tcell inflammatory infiltrate. The authors speculated that the effect of imiquimod may depend on the tumor microenvironment. Nevertheless, it is stated that IMQ can promote a pro-immunogenic tumor microenvironment with histological tumor regression based on the evidence of an immune-mediated response.(29) Concerning Vulvar Paget Disease (VPG), there have also been attempts of imiquimod treatment.(30) Linden et al.,(30) have already performed a twenty patient study following treatment with $5 \%$ IMQ, topically, three times per week over a 16-week period.

There have also been interesting attempts to utilize IMQ as a vaccine adjuvant. Immunization of guinea-pigs with Herpes Simple Virus glycoprotein and imiquimod, reduced effectively virus recurrence in comparison with unimmunized controls.(31) In particular, even though the mechanism of action was not identified, the results indicated that the use of IMQ as an enhancer significantly diminished recurrent lesion days by 5369\%.31 The effect of IMQ has also been examined against Influenza Virus, where Can Li et al.32 focused on the combination of imiquimod with H1N1/415742Md influenza virus particle. Specifically, a compound of IMQ with the virus (50 $\mu \mathrm{g} / 10 \mu \mathrm{g}$, respectively) were intraperitoneally administered in mice, whereas other treatment schemes were utilized as controls (such as, IMQ only group and H1N1 influenze only group). An upregulation of cytokines expressions was noted in both Th-1, Th-2 cytokines with elevated IL-10 secretion levels induced in the IMQ only group, while in the virus only group cytokine storm with increased levels of pro-inflammatory cytokines (IL-6, IFN- $\gamma$, IL-2, IL-4 and IL5) was observed. The outcome revealed that the combination (IMQ and virus group) induced much stronger B cell responses to proliferate and differentiate into antigen specific IgM and IgG secreting antibodies with viral neutralizing activity. The main result was that imiquimod integrated with vaccine antigen can advance potent B cell activation and differentiation leading to accelerated viral specific antibody production, which contribute to the protection against imminent incoming pathogen.(32) In an another novel research, imiquimod has been administrated systemically in order to be tested as an adjuvant that improves immunog enicity of a tumor-lysate vaccine, inducing the rejection of a highly aggressive T-cell lymphoma. The results indicate that T umor cell lysate vaccination using imiquimod as an adjuvant, enhanced the protection from tumor growth and induced a Th1-type as well as humoral immune responses ag ainst LBC cells. The research concluded that imiquimod administered alone significantly enhanced immune response to a tumor lysate vaccine and produced an elevated number of CD4+ 
T-cells and an IFN- $\gamma$ Th1-type response along with specific antibodies.

Imiquimod in clinical oral availability

Imiquimod, a TLR7/8 agonist that induces a potent anti-viral response, is characterized by the production of type I interferons (IFN), pro-inflammatory cytokines and chemokines.(32) A growing literature clearly points a systemic response following topical imiquimod treatment. The tissue response to Aldara treatment across a detailed timecourse model determined the most likely mechanism driving systemic inflammation.(32) It has been shown that topical Aldara treatment induced a potent chemokine and cytokine response throughout the peripheral tissues and brain, with these responses being temporally distinct. IMQ binded to TLR7 on inflammatory cells, such as the Langerhans cells of the epidermis, dendritic cells and monocytes and induced cytokines secretion (IFN- $\alpha$ and TNF- $\alpha$ ). Specifically, the results also presented that IMQ was present in both plasma and brain as early as 4 hours after treatment, suggesting that the primary mechanism of immune activation of topical Aldara treatment was through the direct ligation of IMQ with TLR7 receptors throughout the body.(32) IMQ treatment could induce unintended medium grade systemic side effects that ranged in severity and frequency, including fever, fatigue, headaches, erythema, myalgia, application site inconvenience and raised erythrocyte sedimentation rate.

According to a clinical pharmacokinetic report conducted in order to assess the effect of food on the oral IMQ absorption, to characterize its pharmacokinetics, and to estimate its oral bioavailability on individuals that received a $100 \mathrm{mg}$ oral dose of IMQ, the oral bioavailability was near $47 \%$, with an absorption half-life of close to 1 hour and independent of food consumption. The study suggested that food provided no effect on the rate, extent of absorption or bioavailability of oral imiquimod, and proved the suitability of IMQ for oral administration. In a phase I clinical study, tolerability, toxicity and biological effects of daily oral imiquimod administration were investigated in 21 patients with refractory cancer.(33) Patients were treated with doses of $25 \mathrm{mg}, 50 \mathrm{mg}, 100 \mathrm{mg}$ or $200 \mathrm{mg}$ on a projected 112 day course, in which only three patients completed the course, all at the $50 \mathrm{mg}$ dose. Treatment toxicities were dose related and mainly comprised flu-like symptoms, nausea and lymphopenia. Interferon production was not demonstrated within the first $24 \mathrm{~h}$ of the initial dose but, following repeated doses, ten of the patients developed detectable serum interferon concentrations with a maximum value of $5600 \mathrm{IU} \mathrm{ml}$ recorded. Daily oral administration of imiquimod presented dose- 
depented activation of the interferon production system but at higher doses resulted in flu-like side effect.(33)

\section{PROBLEM VS SOLUTION CONCISE PRESENTATION}

In coronavirus influenzas the ability of the viruses to escape immune surveillance may lead to increased pathogenicity, as an outcome of lymphocytopenia and cytokine storm syndrome. The severity of all types of CoVs is highly related to elevated levels of leukocytes, pro-inflammatory cytokines and desregulation of type I IFN. TLR-7 and 8 play a significant role and in cooperation with PRRs represent the first line of defence against viruse infections. The RNA viruses after infection of host cells trigger signaling cascades resulting in the upregulated secretion of pro-iflammatory mediatora and Type I IFN, which render cells' resistance to CoVs infections. Imiquimod (IMQ) is an immune response modifier that induces immune reactions and the secretion of cytokines reulting in antiviral and antiinflammatory responses. Imiquimod exerts its action in innate immune system by binding to cell surface receptors, especially TLR-7 and TLR-8. The action of Imiquimod to stimulate innate immunity indicates its potential to treat viral infections. We have clear evidence that Imiquimod is able to offer satisfactory stimulation of innate and acquired immunity, helping the elimination of SARS-CoV-2, at least during the early phases of infection.

\section{REFERENCES}

[1]. LiX, Geng M, Peng Y, Meng L, Lu S. Molecular immune pathogenesis and diagnosis of COVID-19. J. Pharm. Anal. (2020) https://doi.org/10.1016/j.jpha.2020.03.001

[2]. Huang C, Wang Y, Li X, Ren L, Zhao J, Hu Y, Zhang L, Fan G, Xu J, Cu X, Cheng Z, Yu T, Xia J, Wei Y, Wu W, Xie X, Yin W, Li H, Liu M, Xiao Y, Gao H, Guo L, Xie J, Wang G, Jiang R, Gao Z, Jin Q, Wang J, Cao B. Clinical features of patients infected with 2019 novel coronavirus in Wuhan, China. Lancet (2020), https://doi.org/10.1016/S0140-

6736(20)30183-5

[3]. Raj VS, Mou H, Smits SL, Dekkers DH, Müller MA, Dijkman R, Muth D, Demmers JA, Zaki A, Fouchier RA, Thiel V, Drosten C, Rottier PJ, Osterhaus AD, Bosch BJ, Haagmans

$B L$. Dipeptidyl peptidase 4 is a functional receptor for the emerging human coronavirusEMC. Nature. 2013;495(7440):251. doi: 10.1038/nature12005

[4]. Zhu N, Zhang D, Wang W, Li X, Yang B, Song J, Zhao X, Huang B, Shi W, Lu R, Niu P, Zhan F, Ma X, Wang D, Xu W, Wu G, Gao GF, Tan W. A Novel Coronavirus from Patients 
with Pneumonia in China, 2019. N EngIJ Med. 2020;382:727

doi:10.1056/NEJMoa2001017.

[5]. Fehr AR, Perlman S. (2015) Coronaviruses: An Overview of Their Replication and Pathogenesis. In: Maier H., Bickerton E., Britton P. (eds) Coronaviruses. Methods in Molecular Biology, vol 1282. Humana Press, New York, NY 2015;1282:1

https://doi.org/10.1007/978-1-4939-2438-7_1.

[6]. Knoops K, Kikkert M, van den Worm SHE, Zevenhoven-Dobbe JC, van der Meer Y, Koster AJ, Mommaas AM, Snijder E. SARS-coronavirus replication is supported by a reticulovesicular network of modified endoplasmic reticulum. PLoS Biol. 2008;6() (2008) https://doi.org/10.1371/journal.pbio.0060226.

[7]. Xu Z, Shi L, Wang Y, Zhang J, Huang L, Zhang C, Liu S, Zhao P, Liu H, Zhu L, Tai Y, Bai C, Gao T, Song J, Xia P, Dong J, Zhao J, Wang F-S. Pathological findings of COVID-19 associated with acute respiratory distress syndrome. Lancet Resp. Med. 2020 https://doi.org/10.1016/S2213-2600(20)30076-X.

[8]. Qin C, Zhou L, Hu Z, Zhang S, Yang S, Tao Y, Xie C, Ma K, Shang K, Wang W, Tian D-S. Dysregulation of immune response in patients with COVID-19 in Wuhan, China. Clinical Infectious Diseases, 2020;ciaa248 https://doi.org/10.1093/cid/ciaa248 [9]. Zhang D, Guo R, Lei L, Liu H, Wang Y, Wang Y, Dai T, Zhang T, Lai Y, Wang J, Liu Z, He A, O'Dwyer M, Hu J. COVID-19 infection induces readily detectable morphological and inflammation-related phenotypic changes in peripheral blood monocytes, the severity of which correlate with patient outcome. 2020 https://doi.org/10.1101/2020.03.24.20042655

[10]. Zhang W, Zhao Y, Zhang F, Wang Q, Li T, Liu Z, Wang J, Qin Y, Zhang X, Yan X, Zeng $X$, Zhang $S$. The use of anti-inflammatory drugs in the treatment of people with severe coronavirus disease 2019 (COVID-19): The experience of clinical immunologists from China, Clinical Immunology 2020 https://doi.org/10.1016/j.clim.2020.108393 [11]. L Liu J, Zheng X, Tong Q, Li W, Wang B, Sutter K, Trilling M, Lu M, Dittmer U, Yang D. Overlapping and discrete aspects of the pathology and pathogenesis of the emerging human pathogenic coronaviruses SARS-CoV, MERS-CoV, and 2019-nCoV. J Med Virol. 2020;92:491 doi: 10.1002/jmv.25709.

[12]. Guo Y-R, Cao Q-D, Hong Z-S, T an Y-Y, Chen S-D, Jin H-J, Tan K-S, Wang D-Y Yan Y. The origin, transmission and clinical therapies on coronavirus disease 2019 (COVID-19) outbreak - an update on the status, Military Medical Research 2020;7 https://doi.org/10.1186/s40779-020-00240-0.

[13]. Bussey KA, Brinkmann MM. Strategies for immune evasion by human tumor viruses. Curr Opin Virol. 2018;32:30.

[14]. Li G, Fan Y, Lai Y, Han T, Li Z. Zhou P, Pan P, Wang W, Hu D, Liu X, Zhang Q, Wu J. 
Coronavirus infections and immune responses. Med Virol. 2020:92:424

https://doi.org/10.1002/jmv.25685

[15]. Kikkert M. Innate Immune Evasion by Human Respiratory RNA Viruses. J Innate Immun. 2020;12:4.

[16]. Gupta AK, Browne M, Blubm R. Imiquimod: A review. J. Cutan. Med. and Surg. 2002;6:554 https://doi.org/10.1177/120347540200600607

[17]. Reiter MJ, Testerman TL, Miller RL, Weeks CE, Tomai MA. Cytokine induction in mice by the immunomodulator imiquimod. J Leukoc Biol. 1994;55(2):234. doi:

10.1002/jlb.55.2.234.

[18]. Schön MP, Schön M. Imiquimod: Mode of action. Br J Dermatol. 2007;157:8 doi: 10.1111/j.1365-2133.2007.08265.x.

[19]. Yoon HK, Shim YS, Kim PH, Park SR. The TLR7 agonist imiquimod selectively inhibits IL-4-induced IgE production by suppressing IgG1/IgE class switching and germline $\varepsilon$ transcription through the induction of BCL6 expression in B cells. Cell Immunol. 2019;338:1. doi: 10.1016/j.cellimm.2019.02.006

[20]. Nerurkar L, McColl A, Graham G, Cavanagh J. The systemic response to topical Aldara treatment is mediated through direct TLR7 stimulation as Imiquimod enters the circulation. Sci Rep 2017;7:16570. https://doi.org/10.1038/s41598-017-16707-5

[21]. Fuertes I, Bastida C, Lopez-Cabezas C, Rodriguez-Carunchio L, Ordi J, Mallolas J, Cranston RD, Blanco JL. The effectiveness and tolerability of imiquimod suppositories to treat extensive intra-anal high-grade squamous intraepithelial lesions/warts in HIVinfected individuals. International Journal of ST D \& AIDS. 2019;30(12):1194 https://doi.org/10.1177/0956462419864506

[22]. Papadavid E, Stratigos AJ, Falag as ME. Imiquimod: an immune response modifier in the treatment of precancerous skin lesions and skin cancer. 2007;8(11):1743. https://doi.org/10.1517/14656566.8.11.1743

[23]. To EE, Erlich J, Liong F, Luong R, Liong S, Bozinovski S, Seow HJ, O'Leary لإ, Brooks DA, Vlahos R, Selemidis S. Intranasal and epicutaneous administration of Toll-like receptor 7 (TLR7) agonists provides protection against influenza A virus-induced morbidity in mice. Sci Rep 2019;9:2366 https://doi.org/10.1038/s41598-019-38864-5 [24]. Kaspari M, Gutzmer R, Kaspari T, Kapp A, Brodersen JP. Application of imiquimod by suppositories (anal tampons) efficiently prevents recurrences after ablation of anal canal condyloma. British Journal of Dermatology 2002;147:757.

https://doi.org/10.1046/j.1365-2133.2002.04979.x

[25]. Kreuter A, Brockmeyer NH, Weissenborn SJ, Wafaisade A, Pfister H, Altmeyer P, Wieland U. 5\% Imiquimod Suppositories Decrease the DNA load of intra-anal HPV Types 6 and 11 in HIV-infected men after surgical Ablation of Condylomata Acuminata. Archives 
of Dermatology, 2006;142(2):243 https://doi.org/10.1001/archderm.142.2.243

[26]. Grimm C, Polterauer S, Natter C, Rahhal J, Hefler L, Tempfer C, Heinze G, Stary G, Reinthaller A, Speiser P. Treatment of cervical intraepithelial neoplasia with topical imiquimod: a randomized controlled trial. Obstetrics and gynecology, 2012;120(1):152 https://doi.org/10.1097/AOG.0b013e31825bc6e8

[27]. Torres A, Storey L, Anders M, Miller RL, Bulbulian BJ, Jin J, Raghavan S, Lee J, Slade HB, Birmachu W. Immune-mediated changes in actinic keratosis following topical treatment with imiquimod 5\% cream. Journal of Translational Medicine, 2007;5(7). https://doi.org/10.1186/1479-5876-5-7

[28]. van Seters M, van Beurden M, ten Kate FJ, Beckmann I, Ewing PC, Eijkemans MJ, Kagie MJ, Meijer CJ, Aaronson NK, Kleinjan A, Heijmans-Antonissen C, Zijlstra FJ, Burger MP, Helmerhorst TJ. T reatment of vulvar intraepithelial neoplasia with topical imiquimod. N EngIJ Med. 2008;358(14):1465 doi: 10.1056/NEJMoa072685.

[29]. Adams S, Kozhaya L, Martiniuk F, Meng TC, Chiriboga L, Liebes L, Hochman T, Shuman N, Axelrod D, Speyer J, Novik Y, T iersten A, Goldberg JD, Formenti SC, Bhardwaj N, Unutmaz D, Demaria S. Topical TLR7 agonist imiquimod can induce immunemediated rejection of skin metastases in patients with breast cancer. Clin Cancer Res. 2012;18(24):6748. doi: 10.1158/1078-0432.CCR-12-1149.

[30]. van der Linden M, Meeuwis K, van Hees C, van Dorst E, Bulten J, Bosse T, IntHout J, Boll D, Slangen B, van Seters M, van Beurden M, van Poelgeest M, de Hullu J. The Paget Trial: A Multicenter, Observational Cohort Intervention Study for the Clinical Efficacy, Safety, and Immunological Response of Topical 5\% Imiquimod Cream for Vulvar Paget Disease. JMIR Res Protoc. 2012;6(9):178 doi: 10.2196/resprot.7503

[31]. Gibson SJ, Lindh JM, Riter TR, Gleason RM, Rogers LM, Fuller AE, Oesterich JL, Gorden KB, Qiu X, McKane SW, Noelle RJ, Miller RL, Kedl RM, Fitzgerald-Bocarsly P, Tomai MA, Vasilakos JP. Plasmacytoid dendritic cells produce cytokines and mature in response to the TLR7 agonists, imiquimod and resiquimod, Cell. Immunol. 2002;218(1-2):74 doi: 10.1016/S0008-8749(02)00517-8

[32]. Nerurkar L, McColl A, Graham G, Cavanagh J. The Systemic Response to Topical Aldara Treatment is Mediated Through Direct TLR7 Stimulation as Imiquimod Enters the Circulation, Sci Rep. 2017;7(1):16570. doi: 10.1038/s41598-017-16707-5

[33]. Savage P, Horton V, Moore J, Owens M, Witt P, Gore ME. A phase I clinical trial of imiquimod, an oral interferon inducer, administered daily. Britsh Journal of Cancer. 1996;74(9):1482 doi:10.1038/bjc.1996.569 